\title{
Ländlicher Raum - eigenständiger Lebensraum ohne eigene Grenzen?
}

\section{Ländlicher Raum heute}

Zwölf Jahre nach ihrer Konstituierung beschäftigte sich die Ministerkonferenz für Raumordnung (MKRO) auf ihrer 14. Sitzung am 12. November 1979 zum ersten Mal fast ausschließlich und intensiv mit dem ländlichen Raum, 15 Jahre nach der Verabschiedung des Raumordnungsgesetzes (ROG). Das Ergebnis der Beratungen sind Entschließungen und Stellungnahmen, die - wie es unter anderem heißt «bei entsprechendem Vollzug sich günstig für den ländlichen Raum auswirken können». Damit war für das wichtigste Koordinationsinstrument der Raumordnungspolitik zwischen dem Bund und den Ländern der ländliche Raum endlich zu einem Thema geworden, hielt es auch die MKRO für erforderlich, «die Bedeutung des ländlichen Raumes für die Bundesrepublik Deutschland, seine Vorzüge, aber auch seine besonderen Probleme, grundsätzlich aufzuzeigen und Ziele sowie Instrumente und Maßnahmen für die Entwicklung des ländlichen Raumes zu benennen' 1 ).

Es genügt meines Erachtens der Hinweis darauf, die MKRO habe sich bereits 1968, wieder 1973 und zuletzt 1977 mit den Verdichtungsräumen und seinen Randgebieten, zusammen Ordnungsräume genannt, beschäftigt ${ }^{2)}$, um mit einem gewissen Recht die Frage stellen zu können, welchen Stellenwert der ländliche Raum in den vergangenen eineinhalb Dekaden für die Raumordnungspolitik eigentlich besessen habe. Aus dem Raumordnungsgesetz selber ist eine Begründung für die bevorzugte Behandlung der einen oder anderen Gebietskategorie kaum ableitbar. Signalisieren die Entschließungen über den ländlichen Raum (weitere sind vorgesehen) eine Wende in der Raumordnungspolitik, die in den vergangenen Jahren ihre Hauptaufgabe in einer Gestaltung der Ordnungsräume sah, sich bevorzugt der städtischen Raumordnung widmete, zugunsten einer Politik des Ausgleichs zwischen den räumlichen Strukturen und gemäß dem unverändert beschworenen Grundsatz, die Gleichwertigkeit der Lebensverhältnisse in allen Teilräumen bewahren bzw. wieder herstellen zu wollen? An eine solche Wende ernsthaft glauben zu können, scheint verfrüht. Denn noch deuten alle Zeichen darauf hin, daß die Interessen der einflußreichen Gruppen unserer Gesellschaft sich unverändert einseitig auf die stärkere Förderung der Verdichtungsräume konzentrieren. Nur dort scheinen sich ihre partikularen, überwiegend kurzfristigen Ziele auch in Zukunft leichter und schneller verwirklichen zu lassen. Und staatliche Raumpolitik kommt diesem Verhalten entgegen, weil sie immer noch zu sehr auf diese sehr manifesten Interessen ausgerichtet ist und nur jene berücksichtigt, die sich vernehmlich äußern ${ }^{3}$. Dieser Konzentration von Macht und politischem Einfluß in den Verdichtungsräumen und seinen Folgen für die räumliche Entwicklung des gesamten Staatsgebietes wird aus dem ländlichen Raum bis heute nicht Gleichwertiges entgegengesetzt.

Unabhängig von der Erkenntnis also, daß die Entwicklung des ländlichen Raumes zu einem eigenständigen, alternativen Lebens- und Wirtschaftsraum nach dem Grundsatz der Gleichwertigkeit der Lebensverhältnisse eine starke, energische, vor allem aber eine Politik des langen Atems gegenüber einem mächtigen Trend verlangt, noch schwieriger zu gestalten durch die schwindenden oder gar negativen Wachstumsraten der Wirtschaft und Bevölkerung, wären die Entschließungen der MKRO zunächst einmal dahingehend zu prüfen, ob die konzipierten Ziele, Instrumente und Maßnahmen, also die gesamte Strategie - ihren Vollzug vorausgesetzt - unter den heutigen Bedingungen überhaupt eine Stärkung des ländlichen Raumes zur Folge haben oder letztlich doch nur wieder übergebührlich starke Entwicklungsimpulse in den Ordnungsräumen auslösen werden. Diese Prüfung, so wichtig sie auch ist, soll an dieser Stelle nicht versucht werden, wie auch die ebenso wichtige Diskussion über die Ursachen der geringen Durchsetzungskraft der Raumordnungspolitik ganz allgemein ausgespart bleibt ${ }^{4}$.

Hier soll vielmehr an der einfachen, aber wohl bedeutungsvollen Feststellung angeknüpft werden, $\mathrm{da} ß$ auch in einer Entschließung über den ländlichen Raum jeder Versuch unterbleibt, die als eigenständig anerkannte Gebietskategorie nach den ihr eigentümlichen Merkmalen und charakteristischen Besonderheiten abzugrenzen. Die MKRO beharrt auf der bekannten «Restraum»-Definition früherer Pro-

Hans Essmann, Bertoldstraße 17, D-7800 Freiburg i. Br. 
gramme des Bundes und der Länder, wenn sie davon ausgeht: "Ländlicher Raum im Sinne dieser Entschließung ist das Bundesgebiet, mit Ausnahme der in der Entschließung der Ministerkonferenz für Raumordnung zur Gestaltung der Ordnungsräume ... angesprochenen Räume» ${ }^{5)}$. So sehr das grundsätzliche Festhalten an einer ländlichen Gebietskategorie und die Bejahung ihrer raumordnerischen Relevanz zu begrüßen ist, die ebenso eine Anerkennung der Existenz räumlich bedingter, spezifischer Lebensformen impliziert wie das aktive Eintreten staatlicher Politik für den Erhalt und die Entwicklung dieser unterschiedlichen Raumstrukturen und Lebensformen, so wenig überzeugend ist es dann, einen "Rest» einen alternativen Lebens- und Wirtschaftsraum zu nennen bzw. ihn in dieser Richtung entwickeln zu wollen. Wird da nicht die Chance vertan, schon durch eine entsprechende Abgrenzung das Charakteristische des ländlichen Raumes, das Erhaltenswerte, Entwicklungsfähige und Verbesserungsbedürftige aufzuzeigen, schon bei der Diskussion von Indikatoren und Kennziffern die Bedeutung seiner Funktionen für den Gesamtraum deutlich werden zu lassen?

Abgesehen von diesem offensichtlichen Mangel ist der «Restraum» vorher zitierter Definition für die praktische Raumordnungspolitik ganz einfach zu groß, ein allzu amorphes Gebilde, für welches sich kaum hinlänglich operationale Ziele formulieren und zielführende Strategien konzipieren lassen. Notwendig ist dagegen eine klare Raumabgrenzung, die den Möglichkeiten der Raumordnungspolitik adäquat ist. Dabei ist der ländliche Raum grundsätzlich zu begreifen als einer von zwei oder mehreren alternativen Lebensräumen, groß genug, die typischen unverzichtbaren ländlichen Funktionen für den $\mathrm{Ge}$ samtraum wahrzunehmen, klein genug, seine ländliche Struktur bewahren und einer ausreichend großen Bevölkerung gleichwertige Lebensverhältnisse garantieren zu können.

Beispielhaft konkreter als die MKRO in der Frage der Abgrenzung des ländlichen Raumes wird sich das MKRO-Mitglied Baden-Württemberg verhalten. Im Landesentwicklungsbericht 1979 heißt es dazu: "Mit der Fortschreibung des Landesentwicklungsplans wird die eigenständige Entwicklung des ländlichen Raumes als Lebens- und Wirtschaftsraum zum Entwicklungsziel; der ländliche Raum ist kein 〈Restraum〉, sondern wird selbständig abgegrenzt»6). Diese Abgrenzung könnte dann ein Ergebnis bringen, welches zeigt, daß es neben den Ordnungsräumen und dem ländlichen Raum noch eine (oder mehrere) Gebietskategorie(n) gibt, die durchaus als weitere Alternative(n) vorstellbar ist (sind) mit eigenen Zielen und spezifischen Entwicklungsstrategien.

Anhand eines bis heute in der Regionalwissenschaft wenig benutzten, wiewohl seit längerem für Raum- analysen als geeignet beurteilten methodischen Hilfsmittels7), nämlich der Skalogramm-Analyse, soll im folgenden eine Möglichkeit der eigenständigen Raumabgrenzung exemplarisch dargestellt werden. Vom Verfasser in seiner Arbeit über den ländlichen Raum Österreichs für den gleichen Zweck angewendet, hat sie zu brauchbaren Ergebnissen geführt ${ }^{8)}$.

\section{Abgrenzung des ländlichen Raumes - ein Beispiel}

Der ländliche Raum ist nicht nur für regionalwissenschaftliche Untersuchungen interessant, das heißt eine rein akademische Größe, sondern ebenso für die praktische Raumordnungspolitik eine zentrale Kategorie. Er ist vorstellbar als eine Zusammenfassung oder auch als ein Konglomerat einzelner, eigenständiger ländlicher Teilräume, die weder nur ausgesprochene Notstandsgebiete, nur periphere, schwach strukturierte noch gänzlich dünn besiedelte Gebiete sein müssen. Auch ist ländlicher Raum nicht mit Land- und Forstwirtschaft gleichzusetzen und eine Politik für den ländlichen Raum ist nicht in erster Linie Agrarpolitik. Vielmehr gehören kleinere zentrale Orte, einzelne stärker verdichtete Bereiche ${ }^{9)}$ ebenso dazu, wie zu den Ordnungsräumen Teilgebiete mit ländlicher Struktur. Bei seiner Abgrenzung wird man in der Regel, da es sich um eine strukturelle Raumkategorie handelt, nach dem Homogenitätsprinzip verfahren, das heißt die noch zu bestimmenden Teilräume oder räumlichen Ausgangseinheiten wegen der Gleichartigkeit oder Ähnlichkeit ihrer raumprägenden Strukturmerkmale zu einer größeren, in bezug auf die gesuchte, komplexe Eigenschaftsdimension als weitgehend homogen zu betrachtende Einheit zusammenfügen.

Für das Beispiel einer möglichen Abgrenzung des ländlichen Raumes wird das Land Baden-Württemberg gewählt. Räumliche Ausgangseinheiten sind die Kreise. Da aber das Kreisreformgesetz von 1971 ehemals 72 Stadt- und Landkreise auf 44 neue Kreise (9Stadt-, 35 Landkreise) zusammenschrumpfen ließ, die wegen ihres größeren Zuschnitts gegenüber den alten Kreisen zwangsläufig in sich erheblich heterogener strukturiert sind, wird für das Abgrenzungsverfahren der ursprüngliche Gebietsstand von 1970 zugrunde gelegt. Obendrein stehen für dieses Jahr, dem der letzten Volkszählung (Stichtag: 25. 6. 1970), die verläßlichsten Daten zur Verfügung.

Basis der eigentlichen Analyse sind aber nur 65 Kreise, weil - rein methodischer Überlegungen wegen - die kreisfreien Städte - Ausnahmen sind Stuttgart, mit einem ausreichend großen Umland, und Baden-Baden - mit ihrem gleichnamigen Landkreis zusammen als eine Raumeinheit behandelt werden. Extreme Werte in denjenigen Strukturbereichen, die für die 
spätere Abgrenzung von Bedeutung sind, werden auf diese Weise einander angeglichen. Gleichzeitig werden die Kreise auch hinsichtlich ihrer Fläche besser miteinander vergleichbar.

Gemeinden als räumliche Basis für eine Abgrenzung des ländlichen Raumes zu wählen, ist schon deshalb nicht sinnvoll, weil eine Reihe wichtiger Statistiken nicht bis auf die kommunale Ebene herunterreichen (z. B. Bruttoinlandsprodukt). Zweitens ist die Variation der Flächengröße und Einwohnerzahl zwischen den einzelnen Gemeinden viel größer als zwischen den Kreisen. Drittens nehmen die Kreise in unserem förderativ verfaßten Staat eine bedeutende raumordnende Mittlerfunktion zwischen dem Land und den Gemeinden wahr, indem die Landräte bzw. ihre Behörden durch Verordnungstätigkeit, Verwaltungspraxis und Beaufsichtigung der Gemeindeverwaltungen spezifische raumordnende Tätigkeit entfalten. Kreise sind daher günstige organisatorische Ansatzpunkte für eine auf die ländliche Gebietskategorie bezogene Raumpolitik. Über die Kreise werden zudem vereinzelte Verdichtungen und größere zentrale Orte in den ländlichen Raum mit einbezogen entsprechend dem oben geäußerten Raumverständnis.

\section{Kennziffern als Hilfsmittel}

Methodisches Hilfsmittel der Abgrenzung sind Kennziffern, die in der Form von Verhältniszahlen aus den Daten der amtlichen Statistik gebildet werden. Solche Kennziffern für größere Gebiete (hier: Kreise) zu bilden hat zwar den Nachteil, daß die innerräumlichen Merkmalsgefälle, soweit gegeben, unberücksichtigt bleiben; da wäre es sicherlich regionalwissenschaftlich konsequenter, die Grenzen homogener Räume völlig unabhängig von jeglicher administrativen Ordnung zu suchen. Aber aus rein praktischen Überlegungen ist es einfach notwendig, scharfe und gegebenenfalls starre Grenzen zu ziehen, die sich bei statistischer Arbeitsweise zwangsläufig an den Verwaltungsgrenzen orientieren. Auf der anderen Seite ist es von nicht geringem Vorteil, die so abgegrenzte Gebietskategorie nahtlos in eine gewohnte, ordnungspolitisch bedeutsame Struktur einfügen zu können.

Die schwierige Frage nach dem Umfang und der $\mathrm{Zu}$ sammensetzung eines Kennziffernkatalogs für die Beurteilung der Homogenität raumprägender Strukturen läßt sich letztlich nicht objektiv beantworten. Auswahlkriterien allgemeiner Verbindlichkeit gibt es leider nicht. Sicherlich wird ein einzelner Indikator zur Charakterisierung komplexer räumlicher $\mathrm{Zu}$ stände in der Regel nicht ausreichen, da generell gilt: je umfangreicher die Zahl unterschiedlicher Kennziffern, desto größer die Wahrscheinlichkeit, das Typische eines Raumes vollständig im Sinne der Aufga- benstellung erfassen und Beziehungszusammenhänge und die für die einzelnen Teilräume spezifischen Merkmalskombinationen aufzeigen zu können. Andererseits wird es nicht sinnvoll sein, auch im Blick auf eine möglichst ökonomische Arbeitsweise der Forschung, alle nur irgendwie datenmäßig erfaßten Strukturelemente ohne eine vorhergehende Prüfung ihrer möglichen Brauchbarkeit für ein Abgrenzungsverfahren heranzuziehen. Vielmehr ist ein analytisches Vorgehen angemessen, welches aus einer großen Zahl im Raum wirkender Faktoren zunächst diejenigen auswählt, die für den Charakter der gesuchten Kategorie bestimmend sind. Es wird also eine Vorauswahl getroffen zwischen wichtigen und weniger wichtigen Merkmalen.

Für die Beurteilung eines Raumes, ländlich-strukturiert zu sein, erscheinen acht Kennziffern als besonders wesentlich. Dabei ist grundsätzlich zwischen zwei Gruppen zu unterscheiden. Die eine umfaßt solche Kennziffern, die a priori das Ländliche eines Raumes charakterisieren und die, wenn es um den Erhalt oder die Wiederherstellung ländlicher Räume als alternative Lebens- und Wirtschaftsräume geht, besondere Zielsetzungen der Raumordnungspolitik verlangen, die andere solche, die eine häufige Folge ländlicher Struktur sind, und die, wenn sie bestimmte negative Werte aufweisen, entsprechende Fördermaßnahmen auslösen sollten. Zur ersten Gruppe innerhalb der acht relevanten Kennziffern gehören ohne Zweifel ein hoher Agrarbesatz, eine geringe Bevölkerungsdichte, ein hoher Anteil von Gemeinden unter 2000 Einwohnern und ein niedriger Industriebesatz, zur zweiten ein niedriges Bruttoinlandsprodukt, eine geringe Realsteuerkraft und negative Wanderungs- und Pendlersalden.

\section{Skalogramm-Analyse}

Die Abgrenzung des ländlichen Raumes geht nun in zwei voneinander unabhängigen Schritten vor sich. Mit Hilfe der Skalogramm-Analyse, einem Skalierungsverfahren, wird zunächst versucht, unter den acht vorgenannten Kennziffern und entsprechend den 65 Kreisen eine Rangordnung in bezug auf die Eigenschaft «ländlich» herzustellen. Dieses Skalierungsverfahren auch unter dem Namen "GuttmanSkala» vor allem in der Soziologie und Psychologie sehr geläufig, ist zwar schon seit geraumer Zeit als eine für regionalwissenschaftliche Fragestellungen brauchbare Methode erkannt ${ }^{9}$, aber, wie schon erwähnt, bis heute selten angewandt worden. Dabei ist sie eine reizvolle Alternative zu der sehr einfachen Kennziffernanalyse und methodisch anspruchsvolleren Verfahren, zum Beispiel der Faktorenanalyse. Schließlich ist im Hinblick auf die praktische Verwendbarkeit der Ergebnisse positiv zu bewerten, daß die Skalogramm-Analyse ohne besondere mathema- 
tische Kenntnisse nachvollziehbar ist; auch sind für ihre Anwendung keine grösseren Hilfsmittel (z. B. EDV) notwendig.

Wie viele andere Verfahren zur Bildung von Indizes oder Skalen setzt die Skalogramm-Analyse Eindimensionalität der Komponenten (hier: räumliche Kennziffern) voraus. Des weiteren gehen nicht die realen Werte in die Analyse ein, sondern stellvertretend maßeinheitsfreie, ordinale und somit vergleichbare Zahlen. Diese «neuen» Daten erhält man, indem zunächst für jedes Item bestimmte Wertbereiche festgelegt werden und dann jeder einzelne Wert danach eingestuft wird, ob er hinsichtlich der gesuchten, komplexen Eigenschaft (hier: ländlich) positiv oder negativ zu beurteilen ist. Zur Milderung dieser ziemlich strikten Dichotomisierung in Wirklichkeit zum Teil sehr unterschiedlicher Daten wird zusätzlich ein neutraler Bereich festgelegt, der ausgehend vom Durchschnittswert der einzelnen Komponenten je ein Viertel der Standardabweichung nach oben und unten umfaßt - einschließlich gewisser Rundungen.

Was die Guttman-Skala jedoch von allen anderen Skalierungsverfahren unterscheidet ist das Kumulative ihrer Teilmerkmale. Operational betrachtet ist eine Ordnung der einzelnen Items der Skala nach dem Grad ihres Gewichts (Teilhabe an der Skala bzw. der gesuchten Eigenschaft) unterstellt dergestalt, daß, wie in unserem Fall, in den Kreisen, in denen der Wert einer schwergewichtigen (oder ranghohen) Kennziffer positiv im Sinne von «ländlich» ein- zustufen ist, immer auch die weniger gewichtigen Kennziffern «ländliche» Werte aufweisen (sollten). Die Kennziffern stehen also in einer bestimmten Rangordnung zueinander und diese Rangordnung hat gleichzeitig eine Rangordnung der Kreise zur Folge. Das Ergebnis einer Skalogramm-Analyse macht beide impliziten Rangordnungen sichtbar. Durch praktisches Probieren - zum Beispiel wie hier nach der Cornell-Technik ${ }^{10}$ - wird die Reihenfolge der Kennziffern und Kreise so lange variiert, bis die bestmögliche Annäherung an die Norm des Kumulativen erreicht, die gesuchten Rangordnungen gefunden sind. Da sich in der Realität eine komplexe Eigenschaft (hier: ländlich) nur selten auf einer perfekten eindimensionalen, kumulativen Skala der beschriebenen Art abbilden läßt, wird es in der Regel mehrere Abweichungen (Fehler) von der Norm geben, die man, innerhalb festgelegter Grenzen allerdings, akzeptieren wird.

Grundsätzlich stehen zwei Möglichkeiten zur Verfügung, die Zahl der Fehler zu minimieren. Erstens kann die Definition der Wertbereiche immer wieder leicht verändert werden. Diese Möglichkeit erschöpft sich jedoch schnell, denn schließlich sollen die Wertbereiche ja zu einer merklichen Differenzierung der Kennziffernwerte (hier: positiv, neutral, negativ) beitragen. Zweitens wird man letztlich auf die Verwendung derjenigen Kennziffern verzichten, die sich nicht in die Skala einpassen lassen, das heißt nicht für die Reproduktion der gesuchten Eigenschaft verwendbar sind. In unserem Beispiel gilt dies

Tabelle 1: Kennziffern und Wertbereiche für eine Skalogramm-Analyse der ländlichen Struktur der Kreise BadenWürttembergs (Stand: 1970).

\begin{tabular}{|c|c|c|c|c|}
\hline \multirow[t]{2}{*}{ Nr. } & \multirow[t]{2}{*}{ Kennziffern } & \multicolumn{3}{|l|}{ Wertbereich } \\
\hline & & positiv (2) & neutral (1)* & negativ (0) \\
\hline 1 & $\begin{array}{l}\text { Erwerbstätige } 1970 \text { in der Land- und Forstwirtschaft } \\
\text { je } 1000 \text { Einwohner }\end{array}$ & über 64 & $64-44$ & unter 44 \\
\hline 2 & $\begin{array}{l}\text { Wohnbevölkerung } 1970 \text { in Gemeinden } \\
\text { bis unter } 2000 \text { Einwohner in v. H. }\end{array}$ & über 31,0 & $31,0-20,0$ & unter 18,0 \\
\hline 3 & $\begin{array}{l}\text { Realsteueraufbringungskraft } 1970 \\
\text { je Wohnbevölkerung in DM }\end{array}$ & unter 190 & $190-240$ & über 240 \\
\hline 4 & $\begin{array}{l}\text { Bruttoinlandprodukt } 1970 \text { je Kopf der Wohn- } \\
\text { bevölkerung in DM }\end{array}$ & unter 10400 & $10400-11500$ & über 11500 \\
\hline 5 & $\begin{array}{l}\text { Erwerbstätige im produzierenden Gewerbe } \\
\text { je } 1000 \text { Einwohner }\end{array}$ & unter 214 & $-* *$ & über 214 \\
\hline
\end{tabular}

* Je ein Viertel der Standardabweichung nach oben und unten um den Durchschnitt in Baden-Württemberg einschließlich gewisser Rundungen.

** Die Bildung von nur zwei Wertbereichen ist sinnvoll, da lediglich sieben Kreise im neutralen Bereich liegen.

Quelle: Statistik von Baden-Württemberg. Gemeindestatistik 1970, Band 161, Heft 2 und 5. Das Bruttoinlandprodukt der kreisfreien Städte und Landkreise 1961, 1968 und 1970. Volkswirtschaftliche Gesamtrechnung der Länder, Heft 4. 
für die beiden Kennziffern: Wanderungssaldo (häufig auch in den Kreisen mit großen Städten negativ oder nur gering positiv) und Pendlersaldo (häufig auch in ausgesprochenen ländlichen Kreisen ausgeglichen, da zwar nur wenige Einpendler, aber mangels erreichbarer Arbeitsplätze ebensowenige Auspendler). Die Bevölkerungsdichte scheidet zusätzlich aus dem Verfahren aus, weil sie exakt das gleiche Wertmuster wie der Agrarbesatz aufweist, beide Kennziffern sich gegenseitig substituieren. Tabelle 1 vermittelt eine Übersicht über die Kennziffern, die endgültig in die Analyse eingehen, und ihre Wertbereiche.

Wir sprachen schon davon, daß Abweichungen von der Norm der kumulativen Skalierung akzeptabel sind, solange sie sich innerhalb festgelegter Grenzen bewegen. Als Maßzahl für die Relevanz eines Ergebnisses gilt allgemein der Reproduzierbarkeitskoeffizient ("overall coefficient of reproducibility»), Quotient aus der Summe der Fehler und der aller Kennziffernwerte (hier: 5 Kennziffern $\times 65$ Kreise $=325$ ) subtrahiert von 1 . Sein Wert gibt Auskunft darüber, wie gut es gelungen ist, eine komplexe Eigenschaft mit Hilfe verschiedener Teilmerkmale auf einer Skala abzubilden. Der Reproduzierbarkeitskoeffizient, abgekürzt $K_{R}$, sollte nicht kleiner als $0,85^{11)}$, das heißt die Zahl der Fehler nicht größer als 15 v. H. der Zahl der Werte sein.

Ein zusätzlicher Test für die Aussagefähigkeit der Skalierung ist die Größe des Skalierungskoeffizienten («coefficient of scalability»), abgekürzt $\mathrm{K}_{S}$. Er wird gebildet als Quotient aus der Summe der Fehler und der möglichen Fehler, somit ein Maß für die Effizienz des Verfahrens, Fehler zu minimieren. Subtrahiert von 1 sollte er nicht kleiner als 0,60 sein, das heißt die Fehler im äußersten Fall 40 v. H. der möglichen ausmachen. Als mögliche Fehler gelten dabei alle die Werte einer Kennziffer, die nicht unter den Wertbereich (hier: positiv, neutral oder negativ) fallen, der die meisten Werte umfaßt. Je gleichmäßiger sich also die einzelnen Kennziffernwerte der Kreise auf die drei Wertbereiche, bezogen auf die einzelnen Kennziffern, verteilen, desto größer ist die Zahl möglicher Fehler und desto besser ist auch die Skalierbarkeit der gesuchten Eigenschaft, wenn gleichzeitig die Zahl der tatsächlichen Fehler am Ende klein ist.

\section{Ergebnis der Skalierung und methodische Einwände}

Das Ergebnis der Skalogramm-Analyse ist in Tabelle 2 dargestellt. Sie bedarf einiger Erläuterungen: Die Zahlen 1, 2, 3, 4 und 5 in der Kopfzeile stehen stellvertretend für die fünf verwendeten Kennziffern, die in Tabelle 1 ausführlich beschrieben und gleichlautend numeriert sind. Die Reihenfolge der
Kennziffern stellt die gesuchte Rangordnung (Gewichtung) dar. Demnach hat der Agrarbesatz die größte Teilhabe an der Eigenschaft «ländlich», der Industriebesatz die geringste. Die Zahlen 2, 1, 0 unter jeder Kennziffer stehen für die Wertbereiche (für Kennziffer 5: Industriebesatz sind nur der positive und negative Wertbereich sinnvoll, da auf den neutralen lediglich sieben von 65 Werten entfallen würden). Gleichzeitig haben diese Zahlen die Bedeutung von Bereichsgewichten, mit denen sich für jeden einzelnen Kreis entsprechend der Ausprägung der Kennziffern Punkte errechnen lassen - gewissermaßen zahlenmäßiger Ausdruck für die Stärke seines ländlichen Charakters. Kreise gleicher Punktzahl - sie sind der äußersten rechten Spalte zu entnehmen werden zu Ranggruppen (siehe äußerste linke Spalte) zusammengefaßt.

Die Kreuze, spaltenweise unter jeder Kennziffer angeordnet, geben wieder, zu welchem Wertbereich die Kennziffern der einzelnen Kreise gehören. Kleine horizontale Striche (Trennlinien) in den Kreuzspalten markieren den Übergang zwischen den einzelnen Wertbereichen entsprechend der Norm einer kumulativen Skalierung, nachdem das Optimum einer möglichst geringen Fehlerzahl erreicht worden ist. Fehler sind diejenigen Kreuze, die unterhalb, bezogen auf die positiven, ober- und unterhalb, bezogen auf die neutralen und oberhalb der Trennlinien, bezogen auf die negativen Wertbereiche, liegen.

Die Häufigkeit der Werte und Fehler je Kennziffer und Wertbereich sowie beider Summe sind den letzten beiden Zeilen der Tabelle 2 zu entnehmen. Daraus lassen sich dann Reproduzierbarkeits- und Skalierungskoeffizient errechnen. Beide liegen mit ihren Werten in unserem Beispiel gut oberhalb der Konvention von 0,85 beziehungsweise 0,60 .

Die Begrenztheit der Aussage einer SkalogrammAnalyse ist vor allem darin zu sehen, daß sie nur einen ordinalen Maßstab als Antwort auf die gestellte Frage liefert. Sie gewichtet zwar die einzelnen Kennziffern entsprechend ihrer Teilhabe an der Skala, sagt aber nichts über die Größe dieser Gewichtung aus. Ebenso weist sie den einzelnen Untersuchungseinheiten (hier: den Kreisen) genau die Position auf der Skala an, die der spezifischen Ausprägung der komplexen Eigenschaft in der jeweiligen Einheit (gemessen durch ausgewählte Kennziffern) entspricht, dadurch ist aber gar nichts über die Größe der Unterschiede der Eigenschaft verschiedener Ranggruppen ausgesagt.

Schließlich ist eine Skalierung überhaupt nur dann möglich, wenn für jede Kennziffer Wertbereiche festgelegt werden, deren Bestimmung wegen des Fehlens theoretisch befriedigender Kriterien beziehungsweise objektiver Maßstäbe jedoch problematisch und letzten Endes eigentlich willkürlich ist. Gerade aber der letzte Vorbehalt trifft natürlich auch für kompliziertere, methodisch anspruchsvollere 
Tabelle 2: Rangordnung der Kreise Baden-Württembergs (Stand 1970) hinsichtlich ihrer ländlichen Struktur

\begin{tabular}{|c|c|c|c|c|c|c|c|c|c|c|c|c|c|c|c|c|}
\hline \multirow{3}{*}{ Rang } & \multirow{3}{*}{$\begin{array}{l}\text { Kreise } \\
+ \text { ) Stadt- und Landkreis }\end{array}$} & \multicolumn{14}{|c|}{ Kennziffern und Bereichsgewichte } & \multirow{3}{*}{ Punkte } \\
\hline & & \multicolumn{3}{|c|}{1} & \multicolumn{3}{|c|}{2} & \multicolumn{3}{|c|}{3} & \multicolumn{3}{|c|}{4} & \multicolumn{2}{|c|}{5} & \\
\hline & & 2 & 1 & 0 & 2 & 1 & 0 & 2 & 1 & 0 & 2 & 1 & 0 & 2 & 0 & \\
\hline I & $\begin{array}{l}\text { Buchen } \\
\text { Stockach } \\
\text { Münsingen } \\
\text { Saulgau } \\
\text { Wangen } \\
\text { Ehingen } \\
\text { Crailsheim } \\
\text { Mergentheim } \\
\text { Müllheim } \\
\text { Schwäb.Hall } \\
\text { Bühl } \\
\text { Uberlingen } \\
\text { Tauberbischofsheim } \\
\text { Sigmaringen } \\
\text { Waldshut } \\
\text { Ohringen } \\
\text { Horb } \\
\text { Wolfach } \\
\text { Donaueschingen } \\
\text { Hochschwarzwald }\end{array}$ & $\begin{array}{l}\mathrm{x} \\
\mathrm{x} \\
\mathrm{x} \\
\mathrm{x} \\
\mathrm{x} \\
\mathrm{x} \\
\mathrm{x} \\
\mathrm{x} \\
\mathrm{x} \\
\mathrm{x} \\
\mathrm{x} \\
\mathrm{x} \\
\mathrm{x} \\
\mathrm{x} \\
\mathrm{x} \\
\mathrm{x} \\
\mathrm{x} \\
\mathrm{x} \\
\mathrm{x} \\
\mathrm{x}\end{array}$ & & & $\begin{array}{l}\mathrm{x} \\
\mathrm{x} \\
\mathrm{x} \\
\mathrm{x} \\
\mathrm{x} \\
\mathrm{x} \\
\mathrm{x} \\
\mathrm{x} \\
\mathrm{x} \\
\mathrm{x} \\
\mathrm{x} \\
\mathrm{x} \\
\mathrm{x} \\
\mathrm{x} \\
\mathrm{x} \\
\mathrm{x} \\
\mathrm{x} \\
\mathrm{x} \\
\mathrm{x} \\
\mathrm{x}\end{array}$ & & . & $\begin{array}{l}\mathbf{x} \\
\mathbf{x} \\
\mathbf{x} \\
\mathbf{x} \\
\mathbf{x} \\
\mathbf{x} \\
\mathbf{x} \\
\mathbf{x} \\
\mathbf{x} \\
\mathbf{x} \\
\mathbf{x} \\
\mathbf{x} \\
\mathbf{x} \\
\mathbf{x} \\
\mathbf{x} \\
\mathbf{x} \\
\mathbf{x} \\
\mathbf{x} \\
\mathbf{x} \\
\mathbf{x}\end{array}$ & & . & $\begin{array}{l}\mathrm{x} \\
\mathrm{x} \\
\mathrm{x} \\
\mathrm{x} \\
\mathrm{x} \\
\mathrm{x} \\
\mathrm{x} \\
\mathrm{x} \\
\mathrm{x} \\
\mathrm{x} \\
\mathrm{x} \\
\mathrm{x} \\
\mathrm{x} \\
\mathrm{x} \\
\mathrm{x} \\
\mathrm{x} \\
\mathrm{x} \\
\mathrm{x} \\
\mathrm{x} \\
\mathrm{x}\end{array}$ & & 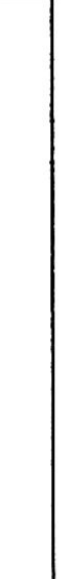 & $\begin{array}{l}x \\
x \\
x \\
x \\
x \\
x \\
x \\
x \\
x \\
x \\
x \\
x \\
x \\
x \\
x \\
x \\
x \\
x \\
x \\
x\end{array}$ & & $\begin{array}{l}10 \\
10 \\
10 \\
10 \\
10 \\
10 \\
10 \\
10 \\
10 \\
10 \\
10 \\
10 \\
10 \\
10 \\
10 \\
10 \\
10 \\
10 \\
10 \\
10\end{array}$ \\
\hline II & $\begin{array}{l}\text { Emmendingen } \\
\text { Sinsheim } \\
\text { Mosbach } \\
\text { Calw }\end{array}$ & & $\begin{array}{l}x \\
x \\
x \\
x\end{array}$ & & $\begin{array}{l}\mathbf{x} \\
\mathbf{x} \\
\mathbf{x} \\
\mathbf{x}\end{array}$ & & & $\begin{array}{l}x \\
x \\
x \\
x\end{array}$ & & & $\begin{array}{l}x \\
x \\
x \\
x\end{array}$ & & & $\begin{array}{l}x \\
x \\
x \\
x\end{array}$ & & $\begin{array}{l}9 \\
9 \\
9 \\
9\end{array}$ \\
\hline III & $\begin{array}{l}\text { Künzelsau } \\
\text { Biberach } \\
\text { Backnang } \\
\text { Tübingen }\end{array}$ & $\begin{array}{l}x \\
x\end{array}$ & $x$ & $\mathbf{x}$ & $\begin{array}{l}\mathbf{x} \\
\mathrm{x} \\
\mathrm{x}\end{array}$ & $\mathbf{x}$ & & $\begin{array}{l}x \\
x \\
x\end{array}$ & $x$ & & $\begin{array}{l}x \\
x \\
x\end{array}$ & $x$ & & $\begin{array}{l}x \\
x \\
x\end{array}$ & $\mathbf{x}$ & $\begin{array}{l}8 \\
8 \\
8 \\
8\end{array}$ \\
\hline IV & $\begin{array}{l}\text { Kehl } \\
\text { Freudenstadt } \\
\text { Ravensburg }\end{array}$ & & $\begin{array}{l}\mathbf{x} \\
\mathbf{x} \\
\mathbf{x}\end{array}$ & & $\begin{array}{l}\mathrm{x} \\
\mathrm{x}\end{array}$ & & $x$ & $\mathrm{x}$ & $\mathbf{x}$ & & $\mathrm{x}$ & $\mathrm{x}$ & & $\begin{array}{l}x \\
x \\
x\end{array}$ & & $\begin{array}{l}7 \\
7 \\
7\end{array}$ \\
\hline $\mathrm{V}$ & $\begin{array}{l}\text { Lahr } \\
\text { Aalen } \\
\text { Offenburg }\end{array}$ & & $\begin{array}{l}\mathbf{x} \\
\mathbf{x} \\
\mathbf{x}\end{array}$ & & & $\begin{array}{l}\vec{x} \\
x\end{array}$ & . & $\begin{array}{l}\mathrm{x} \\
\mathrm{x}\end{array}$ & $\mathbf{x}$ & & $\mathrm{x}$ & & $\begin{array}{l}x \\
x\end{array}$ & $\mathrm{x}$ & $\mathrm{x}$ & $\begin{array}{l}6 \\
6 \\
6\end{array}$ \\
\hline VI & $\begin{array}{l}\text { Vaihingen } \\
\text { Leonberg } \\
\text { Heidelberg } \\
\text { Hechingen } \\
\text { Freiburg } \\
\text { Lörrach }\end{array}$ & & - & $\begin{array}{l}\mathbf{x} \\
\mathbf{x} \\
\mathrm{x} \\
\mathrm{x} \\
\mathrm{x} \\
\mathrm{x}\end{array}$ & $\mathbf{x}$ & $\begin{array}{l}x \\
x \\
x\end{array}$ & $\begin{array}{l}x \\
x\end{array}$ & $\underline{x}$ & $\begin{array}{l}\mathrm{x} \\
\mathrm{x} \\
\mathrm{x} \\
\mathrm{x} \\
\mathrm{x}\end{array}$ & & $\begin{array}{l}\mathbf{x} \\
\mathbf{x} \\
\mathbf{x} \\
\mathbf{x} \\
\end{array}$ & $\begin{array}{l}x \\
x\end{array}$ & 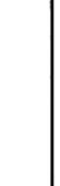 & $\begin{array}{l}\mathrm{x} \\
\mathrm{x} \\
\mathrm{x} \\
\mathrm{x}\end{array}$ & $\begin{array}{l}x \\
x\end{array}$ & $\begin{array}{l}5 \\
5 \\
5 \\
5 \\
5 \\
5\end{array}$ \\
\hline VII & $\begin{array}{l}\text { Konstanz } \\
\text { Bruchsal }\end{array}$ & & & $\begin{array}{l}\mathbf{x} \\
\mathbf{x}\end{array}$ & & & $\begin{array}{l}\mathbf{x} \\
\mathbf{x}\end{array}$ & $\mathbf{x}$ & $\mathbf{x}$ & & $x$ & $x$ & & $\underline{x}$ & $\bar{x}$ & $\begin{array}{l}4 \\
4\end{array}$ \\
\hline VIII & $\begin{array}{l}\text { Rottweil } \\
\text { Säckingën } \\
\text { Tuttlingen } \\
\text { Ulm }{ }^{+} \\
\text {Tettnang } \\
\text { Rastatt }\end{array}$ & & $\begin{array}{l}\mathbf{x} \\
\mathbf{x}\end{array}$ & $\begin{array}{l}\mathbf{x} \\
\mathbf{x} \\
\mathbf{x}\end{array}$ & & $\begin{array}{l}x \\
x \\
x \\
x \\
\end{array}$ & $\begin{array}{l}\mathrm{x} \\
\mathrm{x}\end{array}$ & & $\begin{array}{l}\mathrm{x} \\
\mathrm{x} \\
\mathrm{x} \\
\mathrm{x} \\
\mathrm{x} \\
\mathrm{x}\end{array}$ & & $\mathrm{x}$ & $\begin{array}{l}\mathrm{x} \\
\mathrm{x} \\
\mathrm{x} \\
\mathrm{x}\end{array}$ & $\mathrm{x}$ & & $\begin{array}{l}x \\
x \\
x \\
x \\
x \\
x\end{array}$ & $\begin{array}{l}3 \\
3 \\
3 \\
3 \\
3 \\
3\end{array}$ \\
\hline IX & $\begin{array}{l}\text { Heidenheim } \\
\text { Schwäb.Gmünd } \\
\text { Göppingen } \\
\text { Waiblingen } \\
\text { Nürtingen } \\
\text { Karlsruhe } \\
\text { Baden-Baden }\end{array}$ & & & $\begin{array}{l}\mathrm{x} \\
\mathrm{x} \\
\mathrm{x} \\
\mathrm{x} \\
\mathrm{x} \\
\mathrm{x} \\
\mathrm{x}\end{array}$ & & & $\begin{array}{l}x \\
x \\
x \\
x \\
x \\
x \\
x\end{array}$ & & $\begin{array}{l}\mathbf{x} \\
\mathbf{x} \\
\mathbf{x} \\
\mathrm{x} \\
\mathrm{x} \\
\end{array}$ & $\bar{x}$ & & $\begin{array}{l}\mathrm{x} \\
\mathrm{x} \\
\mathrm{x} \\
\mathrm{x} \\
\mathrm{x} \\
\end{array}$ & $\mathbf{x}$ & $\begin{array}{l}x \\
x\end{array}$ & $\begin{array}{l}x \\
x \\
x \\
x \\
x\end{array}$ & $\begin{array}{l}2 \\
2 \\
2 \\
2 \\
2 \\
2 \\
2\end{array}$ \\
\hline $\mathrm{x}$ & $\begin{array}{l}\text { Villingen } \\
\text { Ludwigsburg } \\
\text { Esslingen } \\
\text { Balingen } \\
\text { Reutlingen }\end{array}$ & & & $\begin{array}{l}x \\
x \\
x \\
x \\
x\end{array}$ & & $\begin{array}{l}x \\
x\end{array}$ & $\begin{array}{l}\mathbf{x} \\
\mathbf{x} \\
\mathbf{x}\end{array}$ & & & $\begin{array}{l}x \\
x \\
x \\
x\end{array}$ & & $\begin{array}{l}x \\
x\end{array}$ & $\begin{array}{l}\mathbf{x} \\
\mathrm{x} \\
\mathrm{x}\end{array}$ & & $\begin{array}{l}x \\
x \\
x \\
x \\
x\end{array}$ & $\begin{array}{l}1 \\
1 \\
1 \\
1 \\
1\end{array}$ \\
\hline$X I$ & $\begin{array}{l}\text { Heilbronn }{ }^{+)} \\
\text {Mannheim } \\
\text { Böblingen } \\
\text { Pforzheim } \\
\text { Stuttgart }\end{array}$ & & & $\begin{array}{l}\mathrm{x} \\
\mathrm{x} \\
\mathrm{x} \\
\mathrm{x} \\
\mathrm{x}\end{array}$ & & & $\begin{array}{l}\mathbf{x} \\
\mathbf{x} \\
\mathbf{x} \\
\mathbf{x} \\
\mathbf{x}\end{array}$ & & & $\begin{array}{l}\mathrm{x} \\
\mathrm{x} \\
\mathrm{x} \\
\mathrm{x} \\
\mathrm{x}\end{array}$ & & & $\begin{array}{l}x \\
x \\
x \\
x \\
x\end{array}$ & & $\begin{array}{l}\dot{x} \\
\mathbf{x} \\
\mathbf{x} \\
\mathbf{x} \\
\mathbf{x}\end{array}$ & $\begin{array}{l}0 \\
0 \\
0 \\
0 \\
0\end{array}$ \\
\hline & Häufigkeit & 22 & 13 & 30 & 31 & 12 & 22 & 33 & 21 & 11 & 35 & 16 & 14 & 39 & 26 & (325) \\
\hline & Fehler & 2 & 2 & 1 & 1 & 3 & 5 & 1 & 4 & 0 & 2 & 4 & 4 & .2 & 4 & $(35)$ \\
\hline
\end{tabular}

$\begin{array}{ll}\text { Reproduzierbarkeitskoeffizient } K_{\mathrm{R}}=89,2 \\ \text { Skalierungskoeffizient }\end{array} \mathrm{K}_{\mathrm{S}}=77,7$ 
Verfahren zu; sie gründen ebenfalls auf ähnlich intersubjektiven Momenten, nur sind diese oft weniger leicht auszumachen.

In diesem Zusammenhang ist ein Hinweis von BOUSTEDT ${ }^{12)}$ beachtenswert, daß Methoden und Instrumente zwar wichtige Hilfsmittel der Interpretation seien, ein endgültiges Urteil aber nicht ersetzen könnten. Darum sei es besonders bei der Entscheidung strittiger Zuordnungsfälle immer noch richtiger, sich dem eigenen Urteilsvermögen oder dem anderer Kundiger anzuvertrauen als die Zuordnung einer statistischen Zufälligkeit zu überlassen.

\section{Ergebnis der Raumabgrenzung}

Weiter oben hieß es "Abgrenzung ... in zwei voneinander unabhängigen Schritten». Ging es beim ersten Schritt lediglich um eine Rangordnung von Kreisen hinsichtlich ihrer ländlichen Struktur, so geht es im zweiten um die eigentliche Raumbegrenzung auf der Basis dieser Rangordnung.

Unzweifelhaft werden die Kreise, die auf der Skala ganz oben stehen (vgl. Tabelle 2), also die besonders ländlichen, als Teile des ländlichen Raumes definiert werden können, während man genauso eindeutig die Kreise ganz unten nicht dazu zählen wird. An die endgültige Zuordnung aller 65 Kreise wird man dagegen die unterschiedlichsten Kriterien anlegen können und deshalb auch zu unterschiedlichen Ergebnissen kommen. Immerhin sind zehn verschiedene Raumaggregate möglich, vorausgesetzt die einzel- nen Ranggruppen werden nicht auseinanderdividiert und die Kreise der Ranggruppe XI (die unterste) in jedem Fall als «nicht-ländlich» klassifiziert.

Im vorliegenden Beispiel wird die mittlere Punktzahl (hier: $\bar{x}=5,9$ ) der Kreise als Ansatz für eine Zäsur gewählt. Kreise mit sechs und mehr Punkten, das sind die Ranggruppen I bis V, bilden zusammen den ländlichen Raum. Berücksichtigt man ferner, daß in Ranggruppe VI bei den Kennziffern 3 und 4 (drei Wertbereiche) Trennlinien den Übergang vom positiven zum neutralen Wertbereich markieren, ließe sich mit einer solchen Begründung auch diese Gruppe in den ländlichen Raum mit einbeziehen. Im Hinblick auf eine den raumordnungspolitischen Möglichkeiten adäquaten ländlichen Gebietskategorie wird die «kleine» Lösung präferiert. Das Ergebnis der Raumabgrenzung ist in Abbildung 1 kartenmäBig dargestellt.

Ein Vergleich von Fläche und Bevölkerung des ländlichen Raumes Baden-Württembergs zwischen der zur Zeit noch gültigen amtlichen Abgrenzung nach der «Restraum»-Definition und der hier vorgeführten (s. Tabelle 3) - im strengen Sinne nicht unmittelbar kompatibel, da die Abgrenzung der Ordnungsräume auf der Basis von Gemeinden bzw. Nahbereichen nicht aber Kreisen vorgenommen wurde - zeigt zwar in der Größenordnung recht deutliche, hinsichtlich der ländlichen Kernräume jedoch keine Unterschiede. Hier besteht weitgehende Identität mit den Gebieten, fürdieschonEntwicklungsprogramme konzipiert wurden ${ }^{14)}$.

Tabelle 3: Fläche und Bevölkerung des ländlichen Raumes Baden-Württembergs im Vergleich zweier unterschiedlicher Abgrenzungsverfahren.

\begin{tabular}{|c|c|c|c|c|c|}
\hline Verfahren & Raumkategorien & $\begin{array}{l}\text { Fläche } \\
\text { abs. }\end{array}$ & $\begin{array}{l}1970 \\
\text { in } \%\end{array}$ & $\begin{array}{l}\text { Bevölkerung } \\
\text { abs. }\end{array}$ & $\begin{array}{l}1970 \\
\text { in } \%\end{array}$ \\
\hline \multirow[t]{2}{*}{$\begin{array}{l}\text { «Restraum»- } \\
\text { Definition }\end{array}$} & $\begin{array}{l}\text { Ordnungsräume } \\
\text { - Verdichtungsräume } \\
\text { - Randzonen }\end{array}$ & $\begin{array}{r}7747,4 \\
2647,7 \\
5099,7\end{array}$ & $\begin{array}{r}21,7 \\
7,4 \\
14,3\end{array}$ & $\begin{array}{r}5127110 \\
3324421 \\
1802689\end{array}$ & $\begin{array}{r}57,7 \\
37,4 \\
20,3\end{array}$ \\
\hline & $\begin{array}{l}\text { Ländlicher Raum } \\
\text { - Verdichtungsbereichel) } \\
\text { - ländlicher Raum }\end{array}$ & $\begin{array}{r}28002,5 \\
2183,7 \\
25818,8\end{array}$ & $\begin{array}{r}78,3 \\
6,1 \\
72,7\end{array}$ & $\begin{array}{r}3767938 \\
1043158 \\
2724780\end{array}$ & $\begin{array}{r}42,3 \\
11,7 \\
30,6\end{array}$ \\
\hline \multirow[t]{2}{*}{$\begin{array}{l}\text { Skalogramm- } \\
\text { Analyse }\end{array}$} & $\begin{array}{l}\text { Nicht-ländlicher Raum } \\
\text { - evtl. ländlicher Raum }\end{array}$ & $\begin{array}{l}14873,8 \\
3044,5\end{array}$ & ${ }_{8,5}^{41,6}$ & $\begin{array}{r}6200441 \\
997340\end{array}$ & ${ }_{11,2}^{69,7}$ \\
\hline & $\begin{array}{l}\text { Lädlicher Raum } \\
\text { (Randgruppen I-V) }\end{array}$ & 20876,1 & 58,4 & 2694607 & 30,3 \\
\hline \multicolumn{2}{|c|}{ Baden-Württemberg, insgesamt } & 35749,9 & 100,0 & 8895042 & 100,0 \\
\hline
\end{tabular}

1) Laut Landesentwicklungsbericht Baden-Württemberg 1979 Teil des ländlichen Raumes s. S. 106.

Quelle: Landesentwicklungsbericht Baden-Württemberg 1975, S. 29. 
Raumabgrenzung :

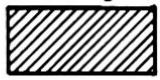

Ländlicher Raum

Pov. noch ländlicher Raum

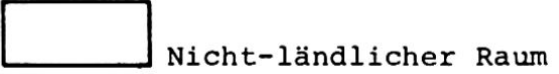

Raumtypen (Kreise):

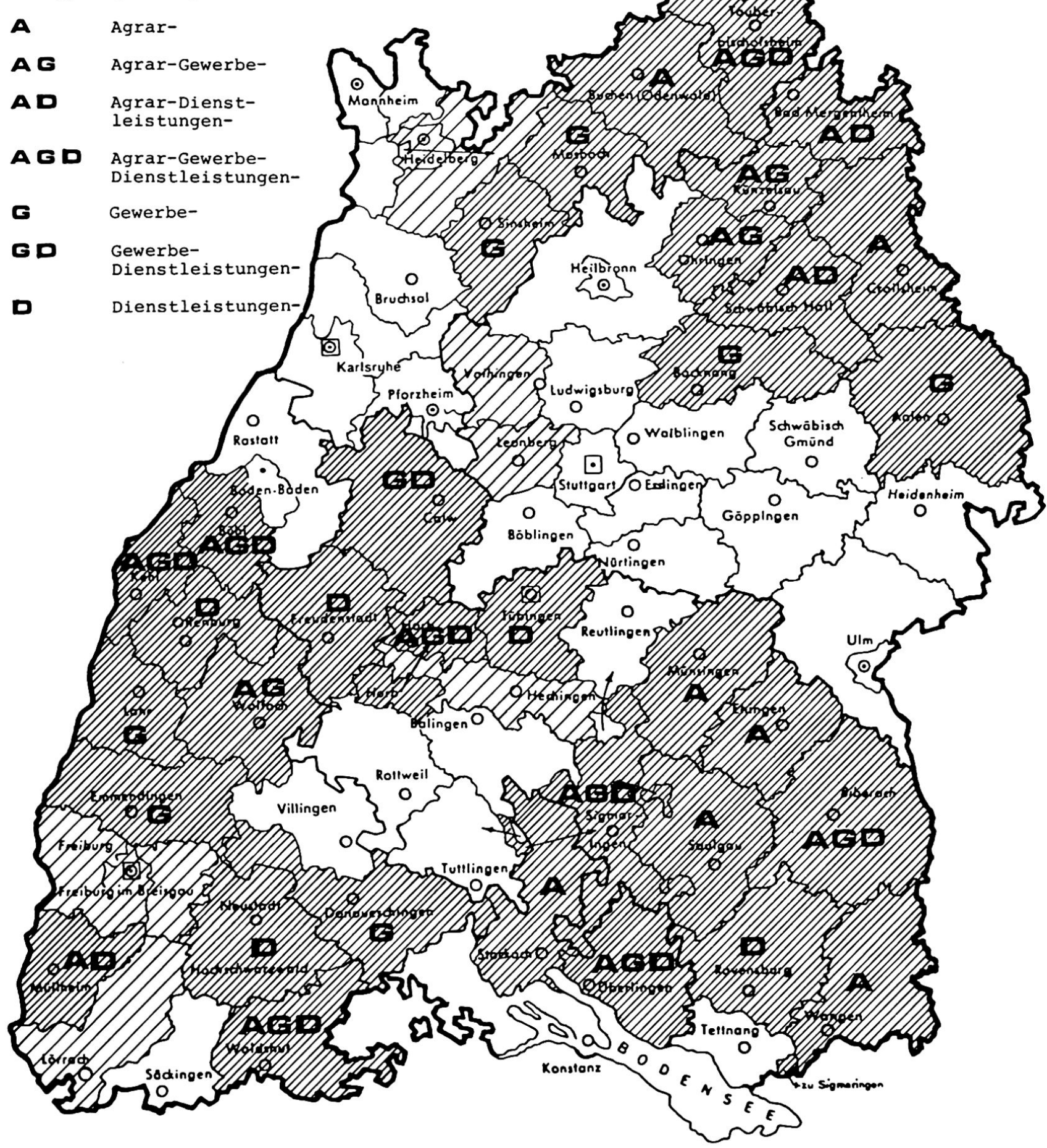

Abb. 1: Der ländliche Raum und ländliche Raumtypen in Baden-Württemberg (Stand: 1970) 


\section{Ländliche Raumtypen}

Der ländliche Raum setzt sich definitionsgemäß aus einzelnen Teilräumen (hier: aus Kreisen) zusammen. Diese weisen sich zwar aufgrund bestimmter Merkmale als ländlich aus, bilden also zusammen eine in diesem Sinne homogene Gebietskategorie, können aber in vielen anderen, raumbedeutsamen Bereichen durchaus unterschiedlich strukturiert sein. Gerade für ein regionalpolitisch differenziertes Vorgehen ist das Herausarbeiten und Darstellen voneinander abweichender räumlicher Gegebenheiten und Entwicklungsmöglichkeiten, die sich dann als Vorzüge oder Probleme beschreiben lassen, ebenso wichtig oder eher noch wichtiger als die Abgrenzung einheitlicher großflächiger Gebietskategorien. Allerdings wird eine sehr detaillierte, ideographische Darstellung für die praktische Raumordnungspolitik wenig Relevanz besitzen, einmal abgesehen davon, daß die Erfassung aller Eigenschaften von Räumen allein schon ein quantitatives Problem ist. Daher erscheint zunächst eine Aggregation einzelner Teilräume zu Raumtypen sinnvoll, deren Verschiedenartigkeit sich anschließend immer noch näher untersuchen läßt. Auf diese Weise wird einmal der Analyseweg erheblich verkürzt, zum anderen die für die Entscheidungsträger so überaus wichtige Aufbereitung und Bündelung vielfältiger Informationen geleistet. Typisierungsverfahren in der regionalen Strukturforschung dienen dem Zweck, räumliche Strukturunterschiede zu charakterisieren, was einen Vergleich an sich unterschiedlicher Raumeinheiten ermöglicht. Vergleichbar sind sie aber erst, wenn sie über eine entsprechende Ordnung oder Gruppierung komparabel gemacht werden. Dazu faßt man die Einzelteile und gleichartigen Merkmalseigenschaften zusammen. Typisierungsverfahren unterstellen, daß sich die Vielfalt der Erfahrungswirklichkeit in den Grundzügen auf wenige Ausgangsvariablen reduzieren läßt. Es genügt demnach, nur die wichtigsten Merkmale als typenbestimmend herauszuarbeiten, so daß auf die weniger wichtigen verzichtet werden kann.

Raumstrukturelle Merkmale entstehen hauptsächlich durch die spezifische Verteilung raumbedeutsamer Aktivitäten der Menschen, mit anderen Worten durch die besondere Anordnung der unterschiedlichen Daseinsfunktionen. Als eines dieser wesentlichen raumprägenden Merkmale kann meines Erachtens die Struktur der Erwerbstätigen am Arbeitsort,

Tabelle 4: Typisierung ländlich-strukturierter Kreise Baden-Württembergs (Stand: 1970) nach Erwerbstätigen am Arbeitsort in der Land- und Forstwirtschaft $(A)$, im produzierenden $(G)$ und Dienstleistungsgewerbe (D)

\begin{tabular}{|c|c|c|c|}
\hline Raumtyp & $\begin{array}{l}\text { Abkür- } \\
\text { zung }\end{array}$ & $\begin{array}{l}\text { Grenzwerte } \\
\text { in } \%\end{array}$ & Kreise \\
\hline Agrarkreis & A & $\begin{array}{l}\text { A: über } 22,0 \\
\text { G: bis } 49,0 \\
\text { D: bis } 34,0\end{array}$ & $\begin{array}{l}\text { Buchen, Crailsheim, Ehingen, } \\
\text { Münsingen, Saulgau, Stockach, } \\
\text { Wangen }\end{array}$ \\
\hline Agrar-Gewerbe-Kreis & $A G$ & $\begin{array}{l}\text { A: über } 17,0 \\
\text { G: über } 49,0 \\
\text { D: bis } 28,9\end{array}$ & $\begin{array}{l}\text { Öhringen, Wolfach, Künzelsau, } \\
\text { Horb, Biberach }\end{array}$ \\
\hline Agrar-Dienstleistungs-Kreis & $\mathrm{AD}$ & $\begin{array}{l}\text { A: über } 17,0 \\
\text { G: bis } 43,9 \\
\text { D: über } 34,0\end{array}$ & $\begin{array}{l}\text { Mergentheim, Müllheim, } \\
\text { Schwäbisch-Hall }\end{array}$ \\
\hline Agrar-Gewerbe-Dienstleistungs-Kreis & AGD & $\begin{array}{l}\text { A: } 12,0-22,0 \\
\text { G: } 44,0-54,0 \\
\text { D: } 29,0-39,0\end{array}$ & $\begin{array}{l}\text { Bühl, Sigmaringen, Tauberbischofs- } \\
\text { heim, Überlingen, Waldshut, Kehl }\end{array}$ \\
\hline Gewerbe-Kreis & G & $\begin{array}{l}\text { A: bis } 17,0 \\
\text { G: über } 55,0 \\
\text { D: bis } 34,0\end{array}$ & $\begin{array}{l}\text { Donaueschingen, Emmendingen, } \\
\text { Mosbach, Sinsheim, Backnang, } \\
\text { Aalen, Lahr }\end{array}$ \\
\hline Gewerbe-Dienstleistungs-Kreis & GD & $\begin{array}{l}\text { A: bis } 11,9 \\
\text { G: über } 49,0 \\
\text { D: über } 34,0\end{array}$ & Calw \\
\hline Dienstleistung-Kreis & $\mathrm{D}$ & $\begin{array}{l}\text { A: bis } 17,0 \\
\text { G: bis } 49,0 \\
\text { D: über } 40,0\end{array}$ & $\begin{array}{l}\text { Hochschwarzwald, Tübingen, } \\
\text { Freudenstadt, Ravensburg, } \\
\text { Offenburg }\end{array}$ \\
\hline
\end{tabular}




\begin{tabular}{|ll|lr|lll|}
\hline \multirow{2}{*}{ Typen } & \multicolumn{3}{|l|}{ Häufigkeit } & \multicolumn{3}{l|}{ Arbeitsbevölkerung } \\
& \multicolumn{2}{|l|}{ abs. in \% } & A & G & D \\
\hline \multirow{2}{*}{ A Agrar-Kreis } & 7 & 20,6 & 26,2 & 44,1 & 29,7 \\
AG Agrar-Gewerbe-Kreis & 5 & 14,7 & 21,4 & 52,5 & 26,1 \\
AD Agrar-Dienstleistungs-Kreis & 3 & 8,8 & 21,2 & 35,4 & 43,4 \\
AGD Agrar-Gewerbe-Dienstleistungs-Kreis & 6 & 17,6 & 18,0 & 47,5 & 34,5 \\
G Gewerbe-Kreis & 7 & 20,6 & 13,5 & 56,9 & 29,5 \\
GD Gewerbe-Dienstleistungs-Kreis & 1 & 3,0 & 11,2 & 49,3 & 39,5 \\
D Dienstleistungs-Kreis & 5 & 14,7 & 11,8 & 44,8 & 43,4 \\
\hline \multirow{2}{*}{ Ländlicher Raum } & \multirow{2}{*}{34} & 100,0 & 17,1 & 48,8 & 34,1 \\
\hline
\end{tabular}

kurz Arbeitsbevölkerung, angesehen werden, die deshalb auch Grundlage einer ersten groben Typisierung ländlicher Teilräume sein soll. Die Arbeitsbevölkerung kann außerdem ganz gut stellvertretend für eine Reihe weiterer prägender Merkmale, wie Siedlungsdichte und -struktur, sozio-ökonomisches Gefüge, Pendlerwesen und anderes mehr, räumliche Zustände zum Ausdruck bringen.

34 Kreise, die insgesamt den ländlichen Raum ausmachen (vgl. Abbildung 1), lassen sich aufgrund des Anteils der drei Wirtschaftssektoren Land- und Forstwirtschaft, produzierendes und Dienstleistungsgewerbe an den Erwerbstätigen am Arbeitsort auf sieben Raumtypen aufteilen: Agrar-Kreis (A), Agrar-Gewerbe-Kreis (AG), Agrar-DienstleistungsKreis (AD), Agrar-Gewerbe-Dienstleistungs-Kreis (AGD), Gewerbe-Kreis (G), Gewerbe-Dienstleistungs-Kreis (GD) und Dienstleistungs-Kreis (D). Abgrenzungskriterium ist die Struktur der Arbeitsbevölkerung im ländlichen Raum, mit anderen Worten, die Mittelwerte aus der Zahl der Erwerbstätigen pro Wirtschaftssektor aller ländlichen Kreise bilden die Grenzen zwischen den einzelnen Typen. Daraus folgt, an einem Beispiel konkretisiert, daß alle Kreise mit überdurchschnittlichem Erwerbstätigenanteil in der Land- und Forstwirtschaft und unterdurchschnittlichem im produzierenden und Dienstleistungsgewerbe als A-Typ bezeichnet werden. Einzige Ausnahme von dieser Regel ist der Mischtyp AGD, der einen Bereich von etwa einem Viertel der Standardabweichung um die Mittelwerte abdeckt, was zur Folge hat, daß die Grenze «Mittelwert» sich für die übrigen sechs Typen um eben diesen Betrag erhöht beziehungsweise erniedrigt ${ }^{15)}$.

Eine Übersicht über die einzelnen Raumtypen, die jeweiligen Grenzwerte und das Ergebnis der Typisierung, nämlich welche Kreise zu welchem Typ gehören, vermittelt Tabelle 4. Dort fällt besonders auf, $\mathrm{da} ß$ nur ein einziger ländlicher Kreis (Calw) als GDTyp eingestuft werden konnte. Insgesamt betrachtet überwiegen erwartungsgemäß die agrarisch gepräg- ten Kreise die übrigen im Verhältnis von 21:13 (s. Tabelle 5). Die Karte in Abbildung 1 zeigt die räumliche Verteilung der einzelnen Typen. Interessant ist hier vor allem ihre Lage zu den Verdichtungsräumen des Landes.

An einem Strukturdreieck (Abbildung 2) wird das Typisierungsraster, welches die einzelnen Raumtypen voneinander abgrenzt, noch einmal bildhaft deutlich. Auf diese Weise läßt sich beispielsweise besonders plastisch darstellen, wie wenig heute extreme Werte die Unterschiede markieren, daß eher Nuancen das Trennende ausmachen. Relativ geringen Differenzen zwischen höchsten und niedrigsten Werten, noch einmal unterteilt durch die Mittelwerte beziehungsweise deren Standardabweichungen, legen die äußerst kleinen Bereiche fest, die dann typenbestimmend sind. Ein Strukturdreieck eignet sich außerdem sehr dafür, den Strukturwandel der Kreise in Stärke und Richtung und damit mögliche Übergänge von einem Raumtyp zum anderen sichtbar zu machen.

Die Signaturen Amin, Amax, Gmin, Gmax, Dmin, Dmax, $\bar{x}_{A}, \bar{x}_{G}$ und $\bar{x}_{D}$ bezeichnen jeweils den höchsten, niedrigsten und durchschnittlichen Anteil der Erwerbstätigen der einzelnen Wirtschaftssektoren in den ländlichen Kreisen Baden-Württembergs.

\section{Abschließende Bemerkungen}

Raumabgrenzungen und die Typisierung von Teilräumen unter Verwendung statistischer Verfahren werden stets unter methodischen Gesichtspunkten diskutiert und kritisch beurteilt werden können. Einmal wird man einen bestimmten Ansatz als irreführend, irrelevant oder einfach als falsch bezeichnen. Oder man wird bei gleicher Ausgangssituation und gleichen oder anderen, vielleicht komplexeren Vorgehensweisen zu mehr oder weniger unterschiedlichen Ergebnissen kommen. Doch bei aller Wertschätzung für die Sorgfalt des Regionalwissen- 
Abb. 2: Typisierung ländlich-strukturierter Kreise Baden-Württembergs (Stand: 1970) nach Erwerbstätigen am Arbeitsort anhand eines Strukturdreiecks

Raumtypen:

A Agrar-Kreis

A $\mathbf{G}$ Agrar-Gewerbe-Kreis

AD Agrar-DienstleistungenKreis

A $\mathbf{Q}$ Agrar-Gewerbe-Dienstleistungen-Kreis

G Gewerbe-Kreis

GD Gewerbe-DienstleistungenKreis

D Dienstleistungen-Kreis

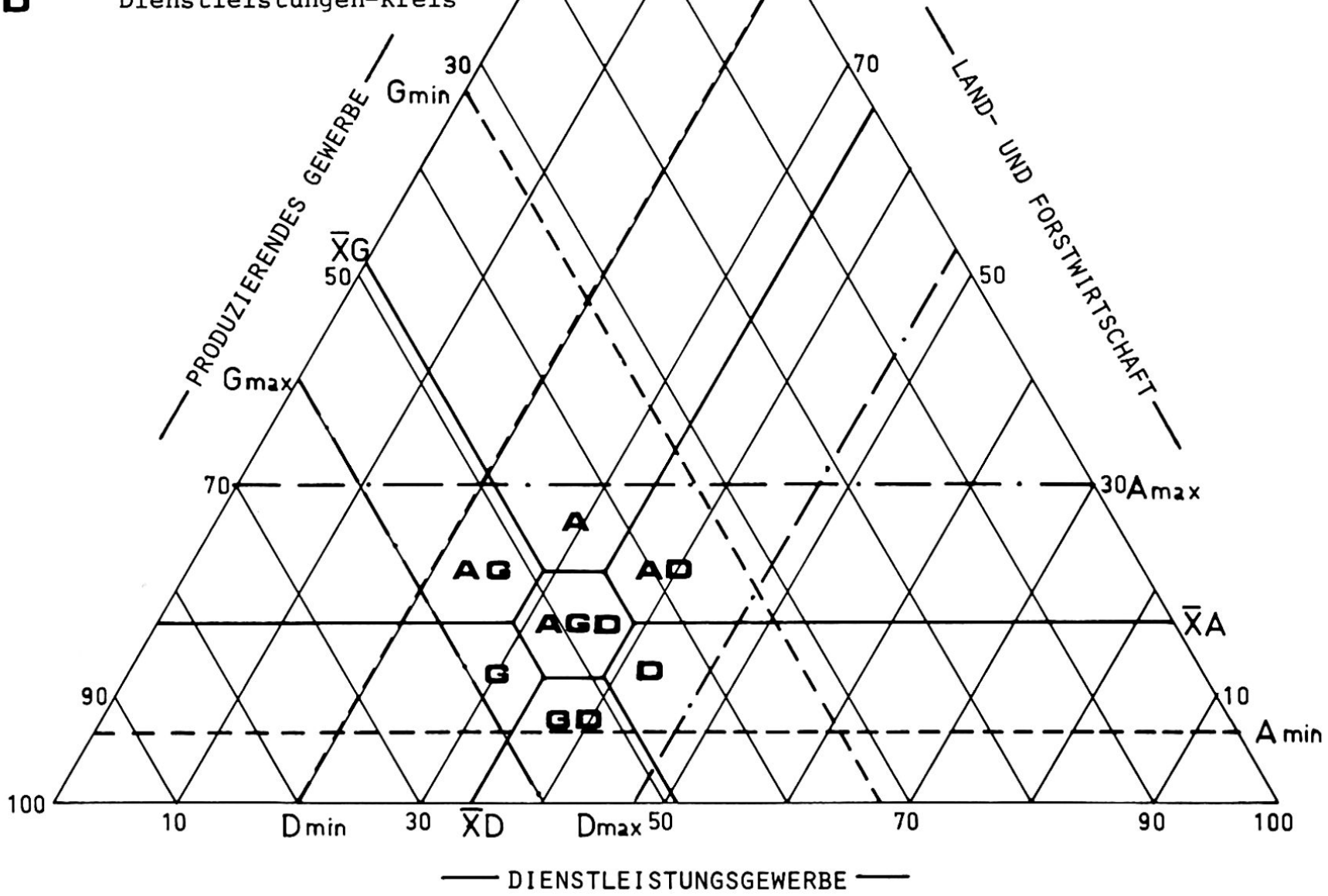

Die Signaturen Amin, Amax, Gmin, Gmax, Dmin, Dmax, $\bar{X} A, \bar{X} G$ und $\bar{X} D$ bezeichnen jeweils den höchsten, niedrigsten und durchschnittlichen Anteile der Erwerbstätigen der einzelnen Wirtschaftssektoren in den ländlichen Kreisen Baden-Württembergs.

schaftlers, die er mit Recht auf die Verwendung geeigneter Methoden und die Suche nach Verbesserungsmöglichkeiten legt, ist nicht zu vergessen, daß Ergebnisse der Raumforschung lediglich ein Hilfsmittel für raumordnungspolitische Entscheidungen sind. Sie dienen der Entscheidungsfindung und sind deshalb wichtige Informationsquellen, haben aber selber nicht schon den Rang einer Entscheidung.
Über die Notwendigkeit von Raumabgrenzungen und -typisierungen für eine rationale staatliche Raumpolitik besteht eigentlich kein Dissens. Deshalb sollte wieder einmal in Erinnerung gerufen werden, daß diese Einsicht auch für den ländlichen Raum gültig sein sollte. An einem Beispiel wurde auch gleich vorgeführt, daß mit relativ einfachen Mitteln brauchbare Ergebnisse zu erzielen sind. 


\section{Anmerkungen}

1) Entschließung der Ministerkonferenz für Raumordnung über den ländlichen Raum vom 12 . November 1979. VZ GStB RP 1980, S. 29 (Heft 3).

2) - Entschließung der Ministerkonferenz für Raumordnung $\mathrm{zu}$ «Fragen der Verdichtungsräume ( $\$ 2$ Abs. 1 Nr. 6 ROG)» vom 21. November 1968 .

- Entschließung der Ministerkonferenz für Raumordnung über das Verhältnis zwischen den Verdichtungsräumen und den anderen Räumen vom 30. Mai 1973.

- Entschließung der Ministerkonferenz für Raumordnung «Gestaltung der Ordnungsräume (Verdichtungsräume und ihre Randgebiete)» vom 31. Oktober 1977.

3) P.ATTESLANDER, Soziologie und Raumplanung. Sammlung Göschen 2110, Berlin/New York 1976, S. 19.

4) Vgl. dazu F. NASCHOLD, Alternative Raumpolitik. Athenäum Verlag Kronsberg/Ts. 1978, S. $14 \mathrm{f}$.

5) Vgl. 1), S. 29.

6) Landesentwicklungsbericht Baden-Württemberg 1979, S. 106.

7) W. ISARD, Methods of Regional Analysis. An Introduction of Regional Science. Cambridge 19675 .

8) H.ESSMANN, Zur Entwicklung des ländlichen Raumes in Österreich. Schriftenreihe des Salzburger Instituts für Raumforschung, Bd. 7, Salzburg 1980.

9) In Baden-Württemberg werden die Verdichtungsbereiche im fortgeschriebenen Landesentwick- lungsplan zum ländlichen Raum gehören, während sie nach dem Landesentwicklungsplan von 1971 zusammen mit den Verdichtungsräumen und den Randzonen zur Kategorie «Verdichtete Räume» gezählt wurden, vgl. Landesentwicklungsbericht Baden-Württemberg 1979, S. 106.

10) Vgl. 7); aber auch J. H. MÜLLER, Methoden zur regionalen Analyse und Prognose. Taschenbücher zur Raumplanung, Bd. 1, Hannover 1973; E. LAUSCHMANN, Grundlagen einer Theorie der Regionalpolitik. Taschenbücher zur Raumplanung, Bd. 1, Hannover 1976.

11) W.J.GOODE and P.K.HATT, Methods in Social Research. New York 1972.

12) R. MAYNTZ, K. HOLZ, P.HÜBNER, Einführung in die Methoden der empirischen Soziologie. Köln/ Opladen 19744 , S. 63.

13) O. BOUSTEDT, Grundriß der empirischen Regionalforschung. Teil I: Raumstrukturen. Taschenbücher zur Raumplanung, Bd.4, Hannover 1975, S. 165.

14) - Albprogramm. Ministerium für Ernährung, Landwirtschaft, Weinbau und Forsten BadenWürttemberg, Stuttgart 1971.

- Schwarzwaldprogramm. Ministerium für Ernährung, Landwirtschaft und Umwelt BadenWürttemberg, Stuttgart 1973.

- Programm für Schwäbisch-Fränkische Gebiete und Odenwald. Wälderprogramm. Ministerium für Ernährung, Landwirtschaft und Umwelt Baden-Württemberg, Stuttgart 1978.

15) Über einen F-Test wurde hohe Signifikanz für die Typisierung ermittelt.
Prof. Dr. Heinrich Rebsamen

Am 8. Juni 1981 starb in Zürich, nahezu 88-jährig, der ehemalige Professor für Geographie am kantonalen Realgymnasium. Anlässlich seines siebzigsten Geburtstages hatte ihn sein Kollege Prof. Dr. Pierre Brunner an dieser Stelle (Bd. 18,1963 , S.224-225) gewürdigt. Hier kann deshalb nur seines Hinschiedes gedacht werden. In den letzten Jahren war es stiller um Prof. Rebsamen geworden. Altersgebrechen suchten ihn heim, so dass der Tod ihm Erlösung bedeutete. Der nachhaltigen Erinnerung aber bleibt wert, was er in seinen 1946 an dieser Stelle veröffent1ichten "Zukunftsaufgaben des Georgraphieunterrichts unserer Mittelschule" festhielt: "Vie1- leicht die vornehmste Aufgabe des gesamten geographischen Unterrichts... besteht darin, die jungen Schweizer mit dem Wesen anderer Kulturen bekannt und vertraut zu machen"... und "Das Suchen nach einer 'Landschaftsethik' darf und soll hier durchaus zur Sprache kommen. Es sind dabei vor allem die Unterschiede zwischen heimischer und fremder Planung hervorzuheben, deren Vergleich wertvolle Impulse für den künftigen Lebensberuf des Gymnasiasten ermöglicht." Wer sich solchen Zielen verpflichtet weiss und sie seinen Jüngern nachlebend weitergibt, kann von der Nachwelt nie vergessen werden. 